\title{
A study on the Effects of Geometrical Parameters of Overlay Coated Layer on the Thermal Stress-strain Distributions of Co-based Super-alloy Deposited Layer on Hot-working Tool Steel
}

\author{
Dae-Seon Guk ${ }^{1}$, Ho-Jin Lee ${ }^{1}$ and Dong-Gyu Ahn ${ }^{1}$ \\ ${ }^{1}$ Chosun University in Gwang-Ju City, Korea
}

\begin{abstract}
Interests in an overlay coating technology, so called hardfacing technology, have steadily increased to improve the service life of hot-working tools through the reduction of the wear of tool surfaces. Characteristics of the overlay coated layer are dependent on geometrical parameters and material properties of sub-layers. The aims of the paper is to examine the effects of geometrical parameters of the overlay coated layer on thermal stress-strain distributions of Co-based super-alloy deposited layer on hot-working tool steel using finite element analysis (FEA). The overlay coated layer is designed as two sub-layers including the wear resistance layer with Co-based super-alloy and the thermal stress control layer (TSCL). The material of the TSCL is composed of $50 \%$ of Co-based super-alloy and $50 \%$ of hot-working tool steel. The protruded height and the inclined angle are chosen as geometrical parameters. The influence of the protruded height and the inclined angle on thermal stress-strain distributions in the vicinity of the overlay coated layer and the strain deviation in joined regions is quantitatively investigated. From the results of the investigation, an appropriate design methodology of the overlay coated layer is discussed.
\end{abstract}

\section{Introduction}

The service life of hot-working tools is greatly shorter than that of the cold-working tools due to the severe wear of the hot-working tools [1]. The premature wear of hotworking tools is induced by the thermal softening of the tool surface and the local bonding of the workpiece to the tool surface [1-3]. The main cause of the thermal softening and the local bonding is the heat transfer from the hot workpiece to the tool surface [1-3].

Numerous researchers have endeavoured to improve the wear resistance of the hot-working tools through an overlay coating technology [3-7]. The overlay coating technology, so called a hardfacing technology, can deposit the super-alloy with excellent wear properties at the elevated temperature on the substrate [3-5]. In addition, the metallurgical bonding is created in the vicinity of the joined region between the overlay coated layer and the substrate [3-5]. Hence, several engineers related to the development of hot-working tools have paid attention to the application of the overlay coating to the hot-working tools [3-5]. Since the metal threedimensional (3D) printing technology has been developed, the interest in the overlay coating technology has greatly increased to extend the service life of hot-working tools through the reduction of an excessive wear of the tool surface $[3,6,7]$.

However, the difference of thermal and mechanical properties between the overlay coated layer and the substrate causes a thermal fatigue phenomenon in the overlay coated layer [8,9]. The thermal stress-strain distribution in the vicinity of the overlay coated layer affects the initiation of the thermal fatigue phenomenon $[8,9]$. The thermal stress-strain distribution in the vicinity of the overlay coated layer is greatly influenced by geometrical parameters of the overlay coated layer.

The aims of the paper is to examine the effects of geometrical parameters, including the protruded height and the inclined angle, of the overlay coated layer on the thermal stress-strain distributions of the Co-based superalloy deposited layer on the hot-working tool steel using finite element analysis (FEA). From the results of FEA, an appropriate design methodology of the overlay coated layer is discussed from viewpoints of the protruded height and the inclined angle.

\section{Finite element analysis}

In order to investigate into thermal stress-strain distributions in the vicinity of the overlay coated layer, heat transfer and thermal stress analyses are performed using an ABAQUS V6.12. Figure 1 illustrates the model of the FE analysis. The axi-symmetrical model with the overlay coated layer is adopted. The overlay coated layer is designed as two sub-layers including the wear resistance layer with Co-based super-alloy and the thermal stress control layer (TSCL). The TSCL is adopted to reduce the thermal stress induced by the 


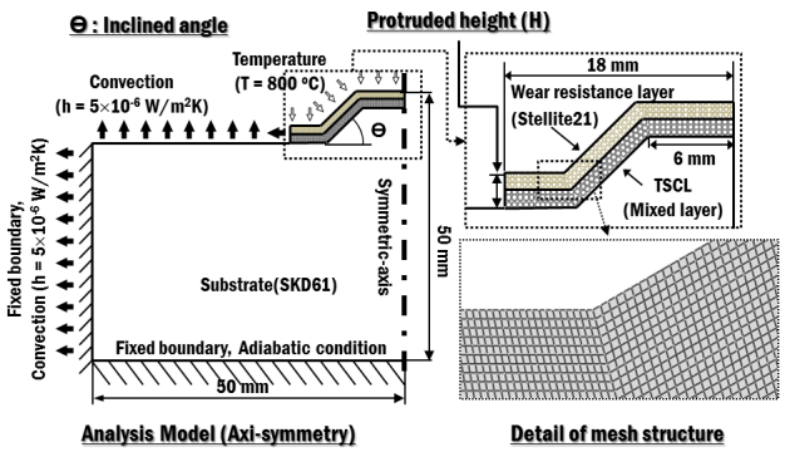

Figure 1. Model of FE analysis.

the wear resistance layer and the substrate [8]. The material of the TSCL is composed of $50 \%$ of Co-based super-alloy and $50 \%$ of the substrate. Stellite 21 and SKD 61 hot-working tool steel are chosen as materials of the wear resistance layer and the substrate, respectively. The thickness of the wear resistance layer is $1 \mathrm{~mm}$. The thickness of the TSCL is identical to that of the wear resistance layer.

In the heat transfer analysis, the constant temperature condition is applied to the top surface of the overlay coated layer. Considering the full annealing temperature of the SKD 61, the applied temperature to the top surface is set to be $800{ }^{\circ} \mathrm{C}[3,6,7]$. The natural convection condition is applied to the top surface exclusive of the overlay coated layer and the side surface. The adiabatic condition is applied to the bottom surface. In the thermal stress analysis, the fixed boundary condition is applied to side and bottom surfaces, as shown in Figure 1. The temperature dependent material properties are applied to the wear resistance layer and the substrates. The density, the thermal expansion coefficient, the elastic modulus, and Poisson's ratio of the TSCL is estimated by the mixing law, as shown in Equation (1) [8]. The specific heat and the thermal conductivity of the TSCL are calculated by Equation (2) and Equation (3), respectively [8]. Materials properties of the Stellite21 and the SKD61

$$
\lambda_{T}=\lambda_{1}(1-\Pi)+\lambda_{2} \Pi
$$

where $\lambda_{\mathrm{T}}, \lambda_{1}, \lambda_{2}$, and $\Pi$ are the property of TSCL, the property of substrate, the property of the overlay coated layer, and the weight fraction of the overlay coated layer, respectively.

$$
\varpi_{T}=\frac{\psi_{1} \varpi_{1}(1-\Pi)+\psi_{2} \varpi_{2} \Pi}{\psi_{1}(1-\Pi)+\psi_{2} \Pi}
$$

where $\varpi_{\mathrm{T}}, \varpi_{1}, \varpi_{2}, \psi_{1}$, and $\psi_{2}$ are the specific heat of TSCL, the specific heat of substrate, the specific heat of the overlay coated layer, the density of substrate, and the density of the overlay coated layer, respectively.

$$
\frac{K_{T}}{K_{1}}=\frac{K_{2}+2 K_{1}+2 \Pi\left(K_{2}-K_{1}\right)}{K_{2}+2 K_{1}-\Pi\left(K_{2}-K_{1}\right)}
$$

where $\kappa_{\mathrm{T}}, \kappa_{1}$ and $\kappa_{2}$ are the thermal conductivity of TSCL, the thermal conductivity of substrate and the thermal conductivity of the overlay coated layer, respectively.
Table 1. Thermal and mechanical properties of Stellite21 and SKD61 at the room temperature [8].

\begin{tabular}{|c|c|c|c|c|c|}
\hline $\begin{array}{c}\text { Mater- } \\
\text { ial }\end{array}$ & $\begin{array}{c}\text { Density } \\
\left(\mathrm{kg} / \mathrm{m}^{3}\right)\end{array}$ & $\begin{array}{c}\text { Thermal } \\
\text { conducti- } \\
\text { vity } \\
\left(\mathrm{W} / \mathrm{m}^{\circ} \mathrm{C}\right)\end{array}$ & $\begin{array}{c}\text { Specific } \\
\text { heat } \\
\left(\mathrm{J} / \mathrm{kg}^{\circ} \mathrm{C}\right)\end{array}$ & $\begin{array}{c}\text { Thermal } \\
\text { expansion } \\
\text { coefficient } \\
\left(10^{-6} / \mathrm{K}\right)\end{array}$ & $\begin{array}{c}\text { Elastic } \\
\text { modulus } \\
(\mathrm{GPa})\end{array}$ \\
\hline $\begin{array}{c}\text { SKD } \\
61\end{array}$ & 7,760 & 25.0 & 460 & 10.4 & 215 \\
\hline $\begin{array}{c}\text { Stellite } \\
21\end{array}$ & 8,330 & 17.0 & 423 & 14.3 & 248 \\
\hline
\end{tabular}

at the room temperature are used to estimate thermal and elastic properties of the TSCL. Table 1 shows thermal and mechanical properties of the Stellite 21 and the SKD61 at the room temperature. The protruded height and the inclined angle are chosen as geometrical parameters of the overlay coated layer, as shown in Figure 1 and Table 2. Table 2 shows ranges of geometrical parameters for FE analyses.

Table 2. Ranges of geometrical parameters for FE analyses.

\begin{tabular}{|c|c|}
\hline Protruded height $\mathbf{( H , ~} \mathbf{m m})$ & Inclined angle $\left(\boldsymbol{\theta},{ }^{\mathbf{o}}\right)$ \\
\hline $0,1,2$ & $0,30,60,90$ \\
\hline
\end{tabular}

\section{Results and discussion}

\subsection{Strain and stress distributions}

Figure 2 shows the influence of the protruded height and the inclined angle on effective stress and major principal strain distributions in the vicinity of the overlay coated layer. Figure 3 represents the variation of maximum values of the effective and stress the major principal strain in the overlay coated layer according to the inclined angle and the protruded height.

Figures 2 (b) and 2 (c) shows that maximum values of the effective stress and the major principal strain occur in the boundary edges. Figures 2 (b) and 3 (a) shows that the maximum value of the effective stress in the vicinity of the overlay coated layer is less than $7.5 \mathrm{MPa}$. The maximum value of the effective stress in the vicinity of the overlay coated layer is negligible irrespective of the protruded height and the inclined angle. The maximum value of the major principal strain is nearly 0.031 , as shown in Figures 2 (c) and 3 (b). From these results, it is noted that the thermal fatigue phenomenon in the vicinity of the overlay coated layer is influenced by the thermal strain distribution.

The maximum value of the major principal strain in the vicinity of the overlay coated layer increases irrespective of the protruded height when the inclined angle augments from $0^{\circ}$ to $60^{\circ}$, as shown in Figures 2 (c)
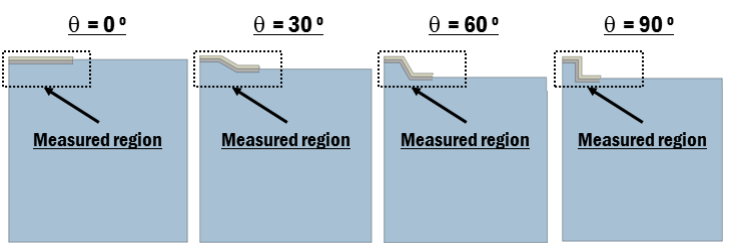

(a) Measured regions 


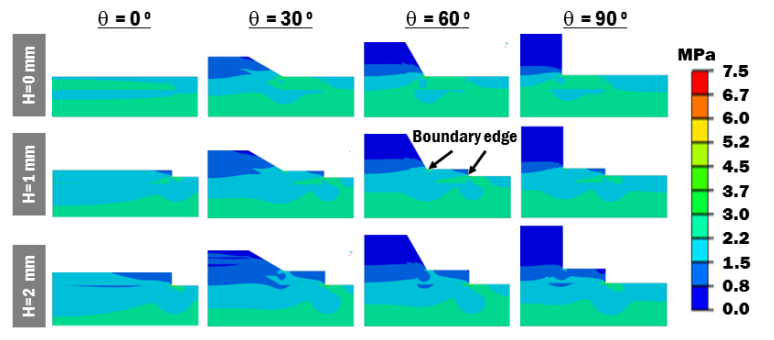

(b) Effective stress

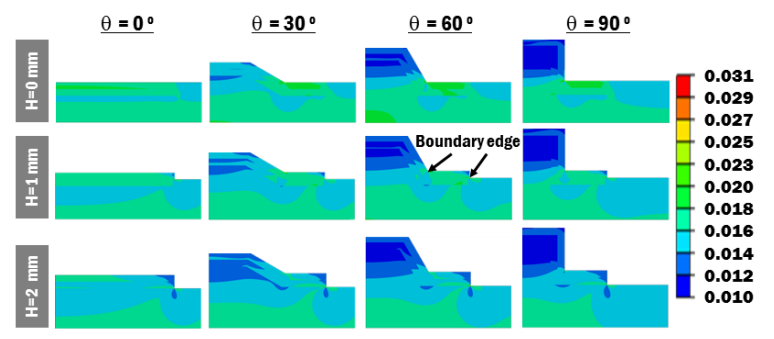

(c) Major principal strain

Figure 2. Influence of the protruded height and the inclined angle on effective stress and major principal strain distributions.

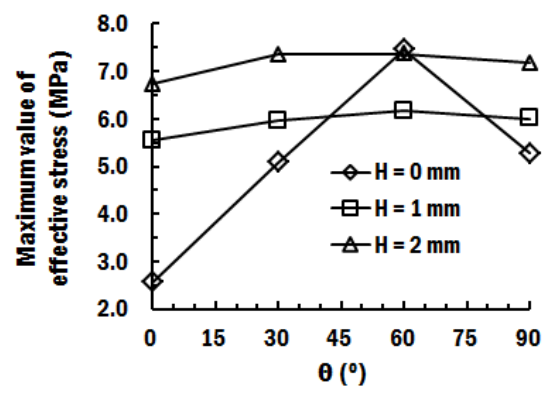

(a) Maximum value of effective stress

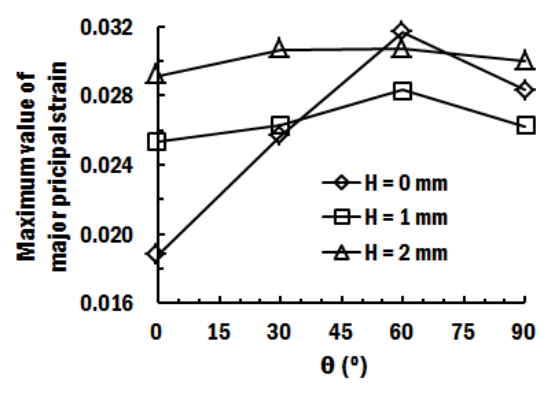

(b) Maximum value of major principal strain

Figure 3. Influence of the protruded height and the inclined angle on maximum values of effective stress and major principal strain.

and 3 (b). In the range of $0^{\circ}-60^{\circ}$ of the inclined angle, the increment of the maximum value of the major principal strain decreases when the protruded height increases. The maximum value of the major principal strain is rapidly changed when the protruded height is 0 $\mathrm{mm}$, as shown Figure 3 (b). The maximum value of the major principal strain in the vicinity of the overlay coated layer decreases when the inclined angle increases from 60 ${ }^{\circ}$ to $90^{\circ}$. The maximum value of the major principal strain augments when the protruded height increases from $1 \mathrm{~mm}$ to $2 \mathrm{~mm}$. From these results, it is revealed that the protruded height and the inclined angle affect the strain distribution in the vicinity of the overlay coated layer.

\subsection{Major principal strain}

The variation of the major principal strain distributions in the depth direction according to different protruded heights and the inclined angles is investigated, as shown in Figure 4. Figure 4 (a) illustrates measured regions and directions of the major principal strain in the vicinity of

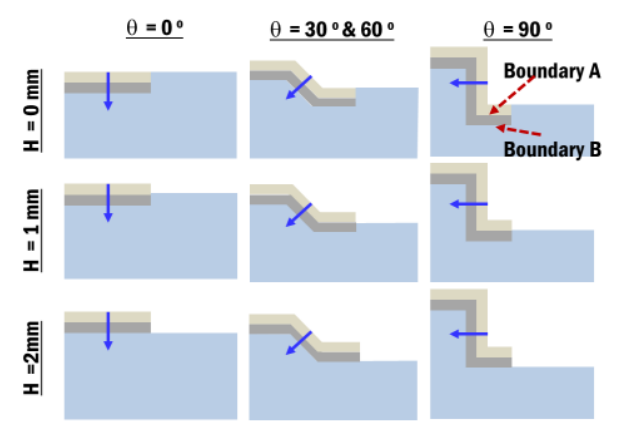

(a) Measured regions and directions

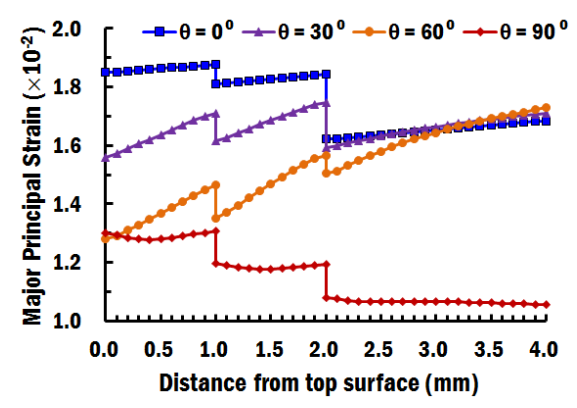

(b) Protruded height $=0 \mathrm{~mm}$

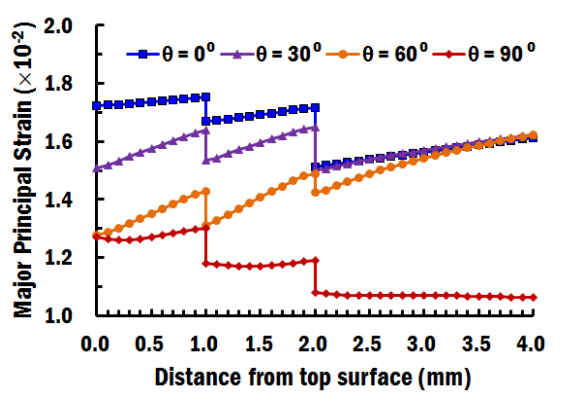

(c) Protruded height $=1 \mathrm{~mm}$

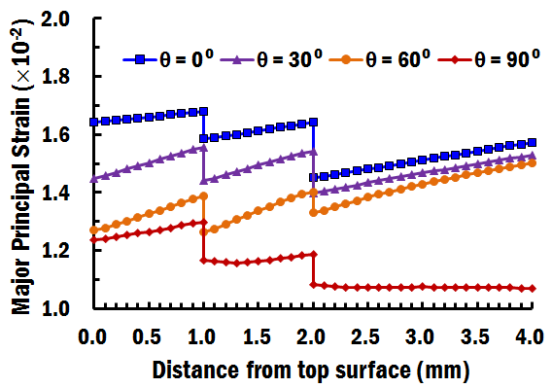

(d) Protruded height $=2 \mathrm{~mm}$

Figure 4. Major principal strain distributions in the depth direction for different inclined angles and protruded heights 
the overlay coated layer. In order to minimize the edge effects in the investigation, the major principal strain is measured in the middle of the overlay coated layer, as shown in Figure 4 (a).

Figures 4 (b), 4 (c) and 4 (d) show that the major principal strains within the wear resistance layer and the TSCL slightly augment when the distance form top surface increases. The major principal strain in the depth direction decreases when the inclined angle and the protrude height increases. The deviation of the major principal strain is clearly found in boundary regions, as shown in Figure 4. Figure 5 shows the estimated deviation of the major principal strain in boundary regions. The Boundary A of Figures 4 (a) and 5 (a) is the border line between the wear resistance layer and the TSCL. The Boundary B of Figures 4 (b) and 5 (b) is the border line between the TSCL and the substrate. The deviation of major principal strain in the Boundary $\mathrm{A}$ almost linearly increases when the inclined angle and the protruded height augment, as shown in Figure 5 (a). However, the deviation of the major principal strain in the Boundary $\mathrm{B}$ decreases when the inclined angle increases from $0^{\circ}$ to $60^{\circ}$ while the of the major principal strain increases when the inclined angle increases from $60^{\circ}$ to $90^{\circ}$, as shown in Figure 5 (b). The deviation of the major principal strain in the Boundary B is greater than that in the Boundary A when the inclined angle is less than nearly $50^{\circ}$. However, the deviation of the major principal strain in the Boundary B is smaller than that in the Boundary A when the inclined angle exceeds $50^{\circ}$. The deviation of the major principal strain in the Boundary B is almost identical to that in the Boundary A when the inclined angle is $90^{\circ}$.

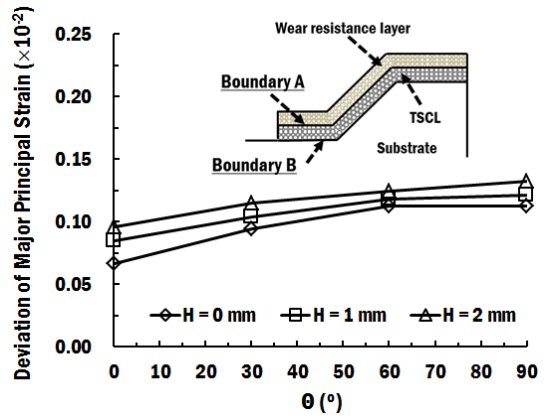

(a) Boundary A

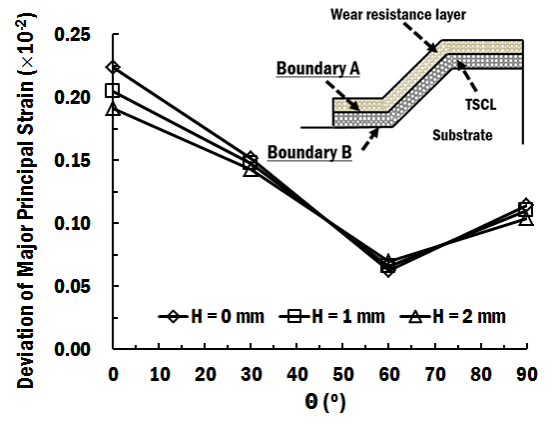

(b) Boundary B

Figure 5. Influence of the protruded height and the inclined angle on the deviation of the major principal strain in the vicinity of the joined region
Based on above results, it is noted that the protruded height should be reduced to decrease the stress, the strain and the strain deviation in the vicinity of boundary edges and joined regions of the overlay coated layer. In order to reduce the strain deviation between the TSCL and the substrate, the strain deviation between the wear resistance layer and the TSCL should be similar to that between the TSCL and the substrate. Hence, it is noted that the flat border line in joined regions should be minimized to decrease the strain deviation between the TSCL and the substrate.

\section{Conclusion}

The effects of the protruded height and the inclined angle of the overlay coated layer on the thermal stress-strain distributions of the Co-based super-alloy deposited layer on the hot-working tool steel using FE analysis. From the results of FE analysis, it has been shown that the protruded height and the inclined angle greatly affect the thermal stress-strain distributions in the vicinity of the overlay coated layer and the deviation of thermal strain in the joined region. In addition, it has been revealed that the thermal fatigue phenomenon in the vicinity of the overlay coated layer is influenced by the thermal strain distribution.

Through the investigation of the influence of the protruded height and the inclined angle on the deviation of the major principal strain in the joined regions, it has been shown that the protruded height should be reduced to decrease the stress, the strain and the strain deviation in the vicinity of boundary edges and joined regions of the overlay coated layer. In addition, it has been revealed that the flat border line in joined regions should be minimized to decrease the strain deviation between the TSCL and the substrate.

\section{References}

1. K. Lange, L. Cser, M. Geiger, J. A. S. Kals, Ann. CIRP. 41, 667 (1992)

2. E. Doege, P. Groche, T. Bobke, Advanced Technology of Plasticity. 1, 27 (1990)

3. D. G. Ahn, Int. J. Precis. Eng. Manuf. 14, 1271 (2013)

4. J. Kohopää, H. Hakonen, S. Kivivuori, Wear 130, 103 (1989)

5. H. Kashani, A. Amadeh, M. Vatanara, Mater. Sci. Technol. 24, 356 (2008)

6. N. R. Park, D. G. Ahn, Int. J. Precis. Eng. Manuf. 15, 941 (2014)

7. N. R. Park, D. G. Ahn, Int. J. Precis. Eng. Manuf. 15, 2549 (2014)

8. N. R. Park, D. G. Ahn, Trans. Korean Soc. Mech. Eng. A. 38, 857 (2014)

9. N. R. Park, D. G. Ahn, Trans. Korean Soc. Mech. Eng. A. 38, 427 (2014) 